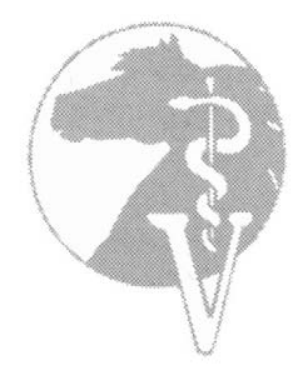

Reviewed

\title{
EQUINE METACARPOPHALANGEAL JOINT SURFACE CONTACT CHANGES DURING WALK, TROT AND GALLOP
}

\author{
J. M. Vilar; M. Pinedo; J. De Mier; F. Castejon; ${ }^{1}$ C. Riber ${ }^{2}$
}

\section{SUMMARY}

Changes in the staining pattern for the metacarpophalangeal joint in the walking, trotting and galloping positions were studied. For this purpose, horse limbs from the third metacarpal bone distally were harvested and placed in the mid-stance position on a hydraulic press that applied the load required to obtain the palmar fetlock angles corresponding to the walking (218 degrees), trotting ( 226 degrees) and galloping positions (240 degrees), and a 1:1000 diluted aqueous dilution of methylene blue was injected into the fetlock joint.

Based on the results, increasing the palmar fetlock angle in the anatomical specimen caused the contact zones of the proximal phalanx and the proximal sesamoidean bones with the third metacarpal bone joint surface to shift dorsally, thereby increasing the contact surface area bctween the involved bones.

In the galloping position, the proximal phalanx was found to extend beyond the dorsal boundary of the third metacarpal bone joint surface and its basal portion to depart from the metacarpus in such a way that the distal sesamoidean ligaments forced the basilar zone of the proximal sesamoidean bones to separate.

These phenomena may obviously assist in explaining the intricate biomechanics of the joint as well as its associated pathologies.

Authors' addresses: 'Departamento de Biologia Animal. Sección Fisiologia. Facultad de Veterinaria. Avda Medina Azahara s/n. Córdoba España. 2 Departamento de Patologia Clinica Veterinaria. Sección Cirugia. Facultad de Veterinaria. Avda Medina Azahara sin. Cordoba España.

\section{INTRODUCTION}

Notwithstanding the dramatic advances in biomechanics in the last twenty years, available knowledge on the etiopathogenesis of major metacarpophalangeal joint pathologies remains inadequate. While chip fractures of the proximal phalanx are ascribed to overstretching, there is no reliable way of justifying why the medial eminence is the most frequently affected. ${ }^{1,2,3}$

On the other hand, proximal sesamoid fractures are assigned to a variety of origins including limb malformation and improper shoeing. ${ }^{1}$ Some authors have suggested that sesamoiditis may predispose fractures of the proximal sesamoids. ${ }^{2,4}$

Denoix ${ }^{5}$ used the contact surface staining method on interphalangeal joints by applying variable random loads and recording any changes observed on photographic plates. Palmer et al ., 6 applied this method to the third carpal bone by using two different loads and proved its usefulness for both biomechanical and pathological studies.

The aim of this work was to expand and improve available knowledge on the function of the metacarpophalangeal joint in the walking, trotting and galloping stance, and accurately define the zones of the involved bones. The results obtained are compared with those for the most common pathologies affecting this joint.

\section{MATERIAL AND METHODS}

Seventy equine forelimbs of healthy slaughtered horses were disarticulated at the carpometacarpal joint, and the specimens deep frozen at $-18^{\circ} \mathrm{C}$ until they were used in this study. 


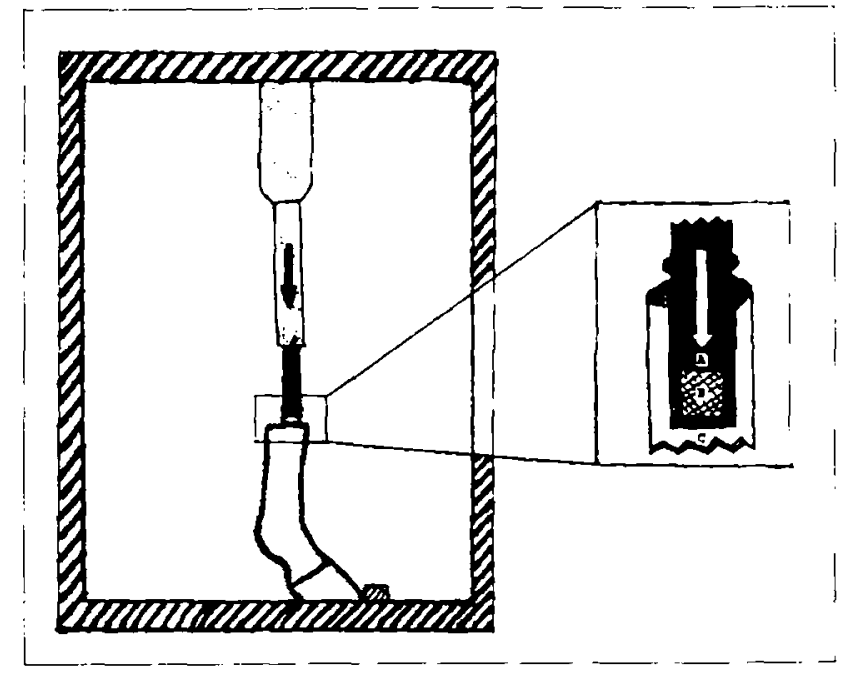

Figure 1. Insertion of the press rod into the medullary cavity of the metacarpus to obtain the anchorage of the specimen. A: Rod. B: Medullary cavity. C: Third metacarpal bone.

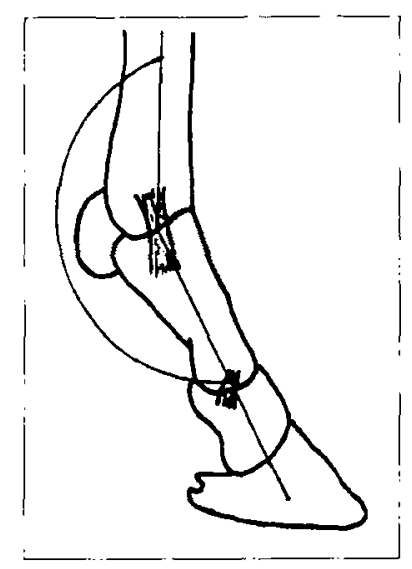

Figure 2. (left) Depiction of the reference lines to allow determination of the required angles.

Ten specimens were used to develop the technique. The remaining sixty limbs were split into three groups of twenty specimens each for walking, trotting and galloping tests, respectively.

A manual hydraulic press-type "jack" (Fertech, Bilbao, Spain) with a maximum applicable load of 3 tons was used for the test.

Once the specimens were thawed, the proximal metaphysis of the third metacarpal bone was cut in order to obtain access to the cancellous bone which allowed drilling by the rod of the hydraulic press. The specimen was positioned under the press and the mechanism of the press was actioned to make the rod enter inside the marrow cavity. Through this the specimen could be effectively stabilized avoiding any unwanted movement (Fig. 1).

After each anatomic specimen was secured on the press, it was oriented in such a way that the third metacarpal bone was positioned perpendicularly to the ground, i.e., in the midstance position. ${ }^{7,8}$ Once the piece was in place, a protractor was fitted to the limb to allow determination of the required angles. For this purpose, two straight refer-

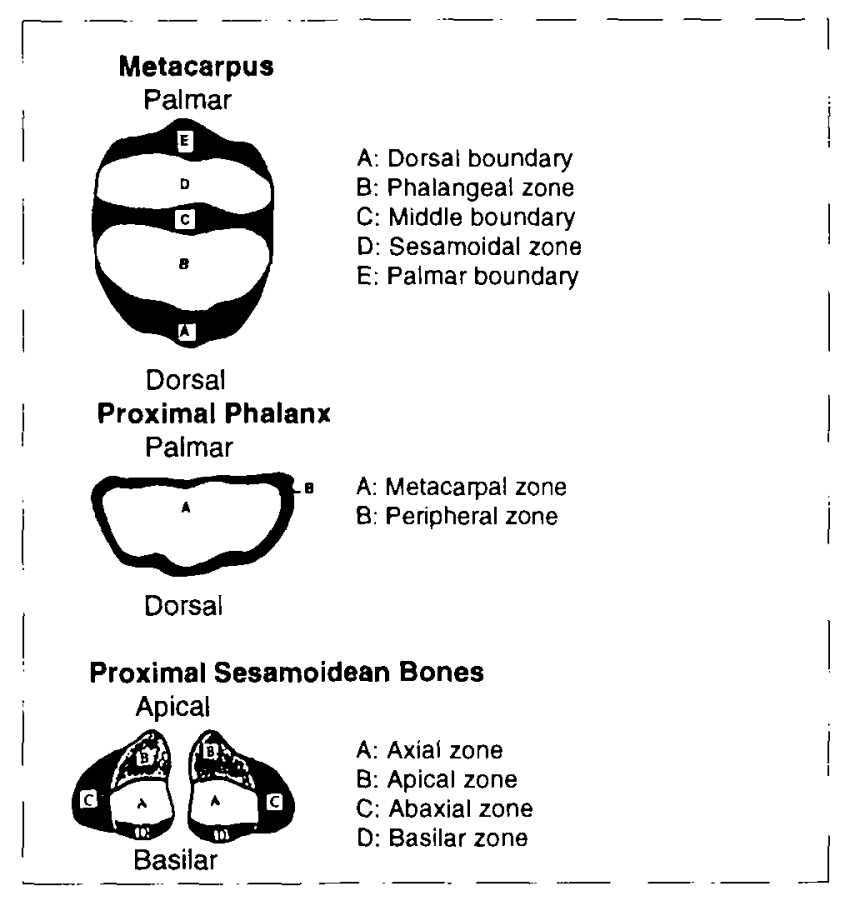

Figure 3. Template zones used to compare staining patterns. The selected nomenclature of the various zones and boundaries is presented.

ence lines were drawn: one represented the long axis of the third metacarpal bone and the other the axis of the proximal phalanx (Fig. 2).

The specimens were then subjected to the load required to obtain the respective palmar fetlock angle corresponding to walking (218 degrees ${ }^{9}$ ), trotting (226 degrees $\left.^{10}\right)$ or galloping $\left(240\right.$ degrees $\left.^{10}\right)$. Once the desired angle was selected, $40-50 \mathrm{ml}$ of a diluted aqueous solution ( $1 \mathrm{~g}: 1000 \mathrm{cc}$ ) of methylene blue was injected into the joint. After $5 \mathrm{~min}$, the time required for the stain to be absorbed, the metacarpophalangeal joints were rapidly dissected and the involved articular surfaces immersed in clean water. Finally, the bones were dried with the help of a hot-air gun used at a prudent distance to avoid the damage of the articular surfaces (maximun temperature: $525^{\circ} \mathrm{C}$ ).

Once each articular component was dry, the soft tissue structures surrounding the joint were carefully dissected in order to facilitate drawing of templates on tracing paper.

Finally, the image analysis program IMAGO was used to determine the surface area of the different contact/noncontact zones of the third metacarpal bone, the proximal phalanx and the proximal sesamoid bones (Fig. 3) observed on the templates and calculated their ratio relative to the overall joint surface area in order to obtain the arithmetic means for all the zones (Tables 1-3).

In order to compare staining patterns, the total articular surface of the bones involved were divided in zones and a nomenclature based on the position of the template zones and bones involved was developed as well (Fig. 3). 
Table 1. Surface areas $(\%)$ of each zone in respect to the entire articular surface of the third metacarpal bone in the three positions studied. Total contact surface areas include full contact and partial contact surface areas.

\begin{tabular}{lccc}
\hline Zone & Walking & Trotting & Galloping \\
Dorsal boundary & 15.34 & 10.50 & - \\
Phalangeal zone & 32.00 & 34.11 & 27.01 \\
Middle boundary & $11.53^{*}$ & 16.00 & 25.28 \\
Sesamoidal zone & 23.00 & 29.46 & 34.11 \\
Palmar boundary & $6.03^{*}$ & 10.02 & 13.60 \\
Total contact & & & \\
Surface area & 37.40 & 49.57 & 61.00 \\
\hline
\end{tabular}

\section{RESULTS}

To facilitate representation of the staining patterns observed on the articular surfaces, a three-color scale was selected: white represented zones in "full contact" that were therefore inaccessible to the stain; grey represented zones that were partially accessible to the stain (such zones had a lighter blue color and were designated "partial contact" zones); and black represented zones where no contact took place. These areas were, therefore, colored deeply blue.

A comparison of the staining patterns for the joint surfaces obtained in the walking, trotting and galloping positions revealed the following:

\section{Metacarpus (Figure 4A and Table 1)}

The dorsal boundary surface area gradually decreased from the walking to the galloping stance, where it eventually disappeared.

The phalangeal zone exhibited a dorsal partial contact subzone and a palmar full contact subzone in the walking position. However, the phalangeal zone was in full contact with the proximal phalanx in the trotting position, and exhibited an increased total contact surface area. In the galloping position, this zone was completely shifted to the dorsal boundary of the metacarpal joint surface and the size of its surface area was decreased relative to the trotting position.

The middle boundary was connected to the palmar boundary in the walking position since the abaxial zones of the proximal sesamoid bones were out of contact in this position, thereby precluding exact calculation of the area of the middle boundary, which could thus only be estimated. In any case, by projecting the contour of the proximal sesamoid bones to their sesamoidal zone of the metacarpal template, then the border between the middle boundary and the sesamoidal zone can precisely be estimated. A comparison of the results for the three positions revealed that
Table 2. Surface areas $(\%)$ of each zone in respect to the entire articular surface of the proximal phalanx in the three positions studied.

\begin{tabular}{lccc} 
& Walking & Trotting & Galloping \\
\hline Metacarpal zone & 74.00 & 78.00 & 72.00 \\
Peripheral zone & 26.00 & 22.00 & 28.00 \\
Total contact & & 78.00 & 72.00 \\
Surface area & 74.00 & & \\
& & & \\
\hline
\end{tabular}

Table 3. Surface areas (\%) of each zone in respect to the entire articular surface of the distal sesamoidean bones in the three positions studied.

\begin{tabular}{lccc} 
Zone & Walking & Trotting & Galloping \\
\hline Abaxial zone & 26.30 & 18.00 & 78.00 \\
Axial zone & 43.70 & 82.00 & \\
Apical zone & 29.00 & & \\
Basilar zone & - & - & 22.00 \\
Total contact & & & \\
Surface area & 43.70 & 82.00 & 78.00 \\
\hline
\end{tabular}

the surface area of the middle boundary increased with increasing palmar fetlock angle.

The sesamoidal zone exhibited a palmar partial contact subzone in the walking position that was abaxially shifted in the trotting position. This zone was in full contact in the galloping position.

The surface area of the palmar boundary increased with increasing palmar fetlock angle, though not to the same extent as the dorsal boundary decreased.

\section{Proximal phalanx (Figure 4B and Table 2)}

The metacarpal zone exhibited two subzones in the walking position: a dorsal of partial contact and a palmar of full contact. However, the entire surface area of the metacarpal zone was in full contact in the trotting position; its peripheral zone was smaller and completely symmetric as in the walking position. Finally, the peripheral zone disappeared in the dorsal region of the bone in the galloping position, while it persisted in the abaxial and palmar regions.

\section{Proximal sesamoid bones (Figure $4 \mathrm{C}$ and Table 3)}

The proximal sesamoid bones showed three distinct zones in the walking position: a non-contact abaxial zone, an apical partial contact zone and an axial full contact zone. However, in the trotting position, the abaxial zone was in partial contact.

Finally, all the zones involved, except the basilar zone which exhibited no contact, were in full contact in the galloping position. 


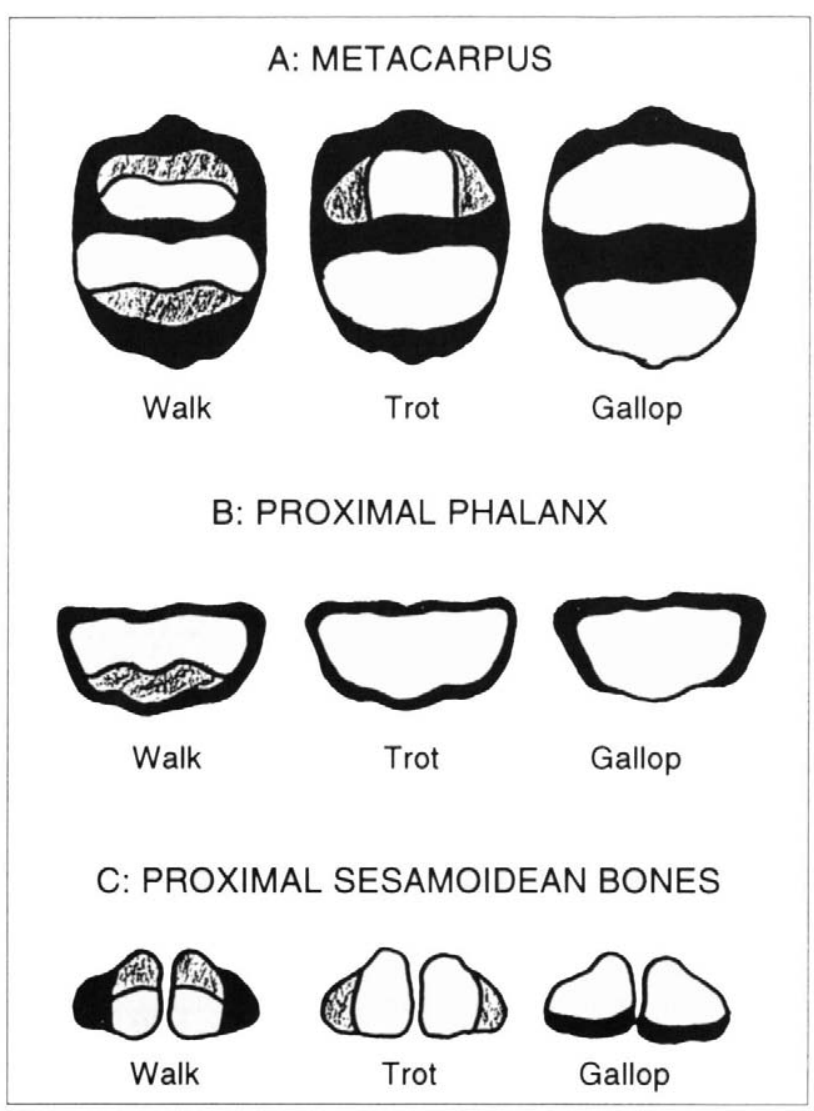

Figure 4. Staining patterns for the joint surfaces of the bones involved in the metacarpophalangeal joint in walking, trotting, and galloping positions.

\section{DISCUSSION}

\section{Metacarpus}

The gradual decrease in the surface area of the dorsal boundary from the walking position to its disappearance in the galloping position indicates that the metacarpus undergoes a net shift relative to the proximal phalanx, thereby causing the phalangeal zone to shift dorsally.

Because the full contact of the phalangeal zone in the walking position is located in the palmar subzone, the principal load is borne by the palmar region of the proximal phalanx. The fact that in the trotting position all the surface area of the phalangeal zone was in full contact suggests that the load is distributed more uniformly over the proximal phalanx. Additionally, because of its increased net surface area, the ability of the joint surfaces to bear an increased load also increases somewhat. The fact that the surface area of this zone is smaller in the galloping position does not contradict the previous statement as it results from the proximal phalanx extending beyond the dorsal boundary of the metacarpal joint surface.

The gradual increase in the surface area of the middle boundary arises from the fact that stress on the

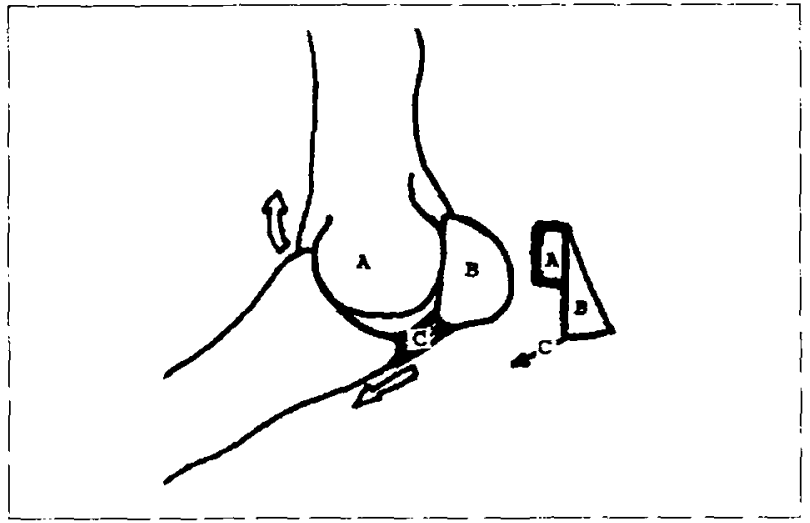

Figure 5. Lever effect at the proximal sesamoid bones. A: third metacarpal bone, $\mathrm{B}$ : proximal sesamoid bones, $\mathrm{C}$ : distal sesamoidean ligaments

metacarpointersesamoidean and suspensory ligaments increases with increasing palmar fetlock angle, which causes a higher distance between the proximal phalanx and proximal sesamoid bones. Therefore, the high stress on the distal sesamoidean ligaments forces them to stretch until they reach the boundary (or even beyond in the galloping position). This suggests that the distal sesamoidean ligaments are highly predisposed to desmopathies such as swelling and fibrillar rupture.

Respecting the sesamoidal zone, at the walking position there is a partial contact at the palmar subzone. This occurs because the distal sesamoidean ligaments are stretched by the dorsal shift of the proximal phalanx, when the joint is extended.

These ligaments have the maximun influence on the proximal sesamoid bones at the walking position, producing full contact on the dorsal subzone; but in the trotting position this partial palmar contact subzone is shifted abaxially making it clear that the metacarpointersesamoidean and suspensory ligaments proximally and the distal sesamoidean ligaments distally are the ones that have most influence on the location and size of the contact areas in this pace.

In the galloping position full contact can be seen in the entire zone.

The surface area of the palmar boundary increases with increasing palmar joint angle, but it does not enlarge at the same rate as the dorsal boundary decreases. This indicates that the proximal sesamoid bones do not follow the dorsal shift of the proximal phalanx because the metacarpointersesamoidean and suspensory ligaments tightly retain these bones allowing very little movement towards the dorsal region of the joint. Therefore most of the attenuation results from stretching of the distal sesamoidean ligaments.

\section{Proximal phalanx}

The decreased surface area of the peripheral zone in the trotting relative to the walking position supports our 
assumption that the higher the load (the brisker the pace), the larger the contact surface area.

The disappearance of the peripheral zone in the dorsal region of the proximal phalanx and the dorsal boundary of the metacarpus in the galloping position is the result of the contact between the dorsal edges of both articular surfaces, which in theory may predispose the development of chip fractures.

The abaxial and palmar regions of the peripheral zone exhibit no contact, so the zone is literally detached from the metacarpus. This, as will be explained, has consequences on the proximal sesamoid bones.

\section{Proximal sesamoid bones}

The non-contact area in the abaxial zone in the walking position suggests that the extensor branches of the suspensory ligament play no prominent role in this position.

However, the zone is in partial contact in the trotting position, and hence the extensor branches of the suspensory ligament begin to exert their effect.

The non-contact basilar zone of the proximal sesamoid bones in the galloping position develops as follows: as the palmar portion of the proximal phalanx separates from the metacarpus, due to the traction exerted by the distal sesamoidean ligaments, the sesamoidal base detaches from the metacarpus. It is our belief that this results in a lever effect at the proximal sesamoid bones, so their basilar portions are in theory highly prone to fracturing, particularly at the lever axis (Fig. 5). In this respect, some authors ${ }^{1.3}$ claim that these bones tend to fracture at the apex rather than the base. On the other hand, Sandoval and Aguera, ${ }^{11}$ believe that racehorses are specially prone to basilar fractures. However, the clinical practice shows that apical fractures are more frequently encountered, perhaps because the apical regions of the proximal sesamoid bones are much thinner than the basilar ones and therefore more prone to fracturing through distraction forces.

The above-mentioned leverage forces are also observed in the apical and abaxial zones of these bones in the walking and trotting positions. Even though the loads borne at both paces are only moderate, they predispose to fractures in the event of a pathologic condition (e.g., in horses with sesamoiditis ${ }^{2,4}$ ).

\section{REFERENCES}

1. Stashak TS: Adams' Lameness in Horses. 4th edition. Philadelphia: Lea \& Febiger, 1987;568-584.

2. Simon A: Traumatologic surgery in large animals. In: Jennings PB, ed. The Practice in Large Animals. Philadelphia: W.B.Saunders Company, 1989;675-832.

3. Richardson DW: Diseases of the fetlock region. In: Colahan P, Mayhew I, Merritt A, Moore J, eds. Equine Medicine and Surgery. 4th edition. California: American Veterinary Publications, 1991;1379-1391.

4. Honnas C: Surgical treatment of selected musculoskeletal disorders of the forelimb. In: Auer J ed. Equine Surgery. Philadelphia: W.B. Saunders Company, 1992;9851055.

5. Denoix JM: Étude biomecanique des articulations interphalangiennes du cheval par le methode des surfaces de contact. 13éme Journeé détude. Alffort. France, 1987.

6. Paimer J, Bertone A, Litsky A: Contact area and pressure distribution changes of the equine third metacarpal bone during loading. Equine Vet $J$ 1994;5:197-202

7. Drevemo S, Dalin G, Fredricson I, Hjerten E: Equine locomotion 1,2,3. Equine Vet $J$ 1980;60-73.

8. Clayton HM: Locomotion. In: Jones W, ed. Equine Sports Medicine. Philadelphia: Lea \& Febiger, 1989;149-185.

9. Miro F, Martinez A, Vivo J, Morales JL, Monterde J: Angulos articulares del caballo español al paso. Salon internacional del caballo. Sevilla. Spain, 1994.

10. Martinez A: Personal communication. 1994.

11. Sandoval J, Aguera E: Anatomia Aplicada Veterinaria. Imprenta moderna. Cordoba. Spain, 1985.

\section{TWO BOOKS ON ALTERNATIVE THERAPIES}

\section{Alternative Medicine: The definitive guide Deepak Chopra, M.D.}

An authoritative text on the many facets of alternative medicine. Written in a style useful to physicians, veterinarians and all others interested in alternative health care for humans and animals. Over 1000 pages hardbound. $\$ 60.00$ US $\$ 70.00$ outside the US

For credit card orders call 909-678-1889.

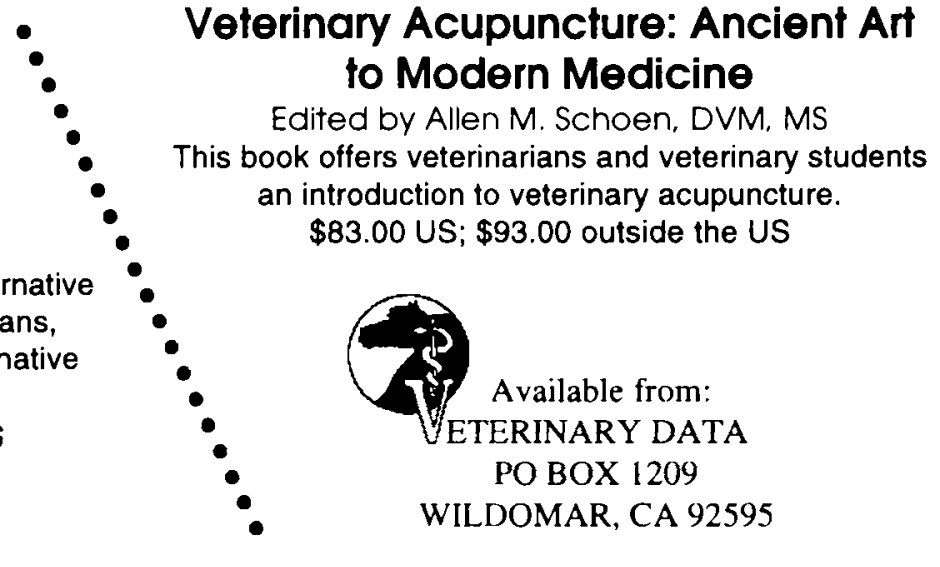

